\title{
Tratamiento óptimo del infarto con elevación del segmento ST: una carrera de obstáculos
}

\author{
Optimal Treatment of ST-segment Elevation Myocardial Infarction: An Obstacle Race
}

MANEL SABATÉ

Desde hace 2 décadas, se ha demostrado el beneficio de la angioplastia primaria sobre la trombólisis para la reperfusión del infarto con elevación del segmento ST (IAMEST). Efectivamente, los diversos estudios clínicos aleatorizados y metaanálisis han demostrado una reducción de la tasa de mortalidad, reinfarto, reoclusión, necesidad de revascularización urgente, accidente vascular cerebral hemorrágico entre otras en comparación con la trombólisis. (1-2) Desde entonces, las guías de práctica clínica consideran este tratamiento invasivo como método de referencia (gold standard) siempre que sea llevado a cabo por un equipo experto, en un centro de excelencia, en los tiempos recomendados (<120 min desde el diagnóstico electrocardiográfico) dentro de una red regional de asistencia del IAMEST. (3) Sin embargo, la implementación de estas recomendaciones no ha sido universal ni uniforme en todos los países del planeta. Hoy en día, existen aún diferencias regionales manifiestas en la atención a los pacientes con IAMEST. Las barreras que se identifican para el desarrollo de estas redes regionales suelen ser comunes (Tabla 1). Globalmente, no se trata de un problema económico, es más, el desarrollo de una red puede llegar a ser coste-eficaz. (4) En este sentido, clásicamente, se han evidenciado tasas de reperfusión con angioplastia primaria muy elevadas en países con renta per cápita menor que la de otros países con rentas más elevadas. (5) Se trata más de conseguir una concienciación a todos los niveles: política, médica y social. Desde el punto de vista político, el código infarto debe pasar a ser una prioridad asistencial. El impulso y liderazgo desde la administración es clave. Esta debe trabajar en coordinación con los servicios de emergencias médicas y los profesionales de los diferentes hospitales y centros de salud implicados en el proceso y circuito del paciente que sufre un IAMEST. Debe diseñarse un protocolo clínico de consenso entre todas las partes y acordarse una sectorización del flujo de pacientes según la localidad donde vive y el hospital de referencia con capacidad para el tratamiento invasivo. Como parte
Tabla 1. Barreras para la implementación de programas de angioplastia primaria

Presencia de sistemas regionales de salud con modelos (priorida-
des) de salud diferentes incluso dentro de un mismo país
Falta de conocimiento general acerca del beneficio de la angio-
plastia primaria como tratamiento que salva vidas en el IAMEST.
No existencia de regulaciones sobre el transporte de pacientes con
IAMEST para angioplastia primaria en la mayoría de regiones.
Apoyo político escaso (poca percepción de esta iniciativa como
de interés para la población).
Presupuesto regional limitado para salud.
Falta de información de la población general sobre los síntomas de
alarma y del procedimiento por realizar en caso de presentar sínto-
mas sospechosos. Número de teléfono de llamada única: p. ej., 112.
Pobre motivación para entrar datos en un registro individual de
pacientes.
Profesionales reacios a iniciar un programa sin apoyo institucional.

esencial, el paciente debe aprender a reconocer las señales de alarma y conocer el dispositivo de activación de emergencia. En este sentido, la realización de campañas de concienciación (6) que deberían irse repitiendo cada cierto tiempo ayudarán a mantener a la población en alerta. Finalmente, y no menos importante, debe registrarse la actividad y los resultados en salud para definir las áreas de mejora y los puntos donde incidir. La red de infarto debe ser dinámica para poder adaptarse a los diferentes cambios que se pueden producir (apertura de nuevos centros, migración de población, aparición de nuevos tratamientos, etc.).

Blanco y colaboradores presentan en el número de la revista (7) la experiencia de un centro de alto volumen durante 20 años en el tratamiento del IAMEST. Han analizado los datos de lo acontecido durante una primera década (2000-2009), en la cual se iden- 
tificaron las barreras para el tratamiento y se fueron implementando mejoras asistenciales que se han visto reflejadas en la segunda (2010-2019). El trabajo médico realizado por el centro es encomiable y merece un gran reconocimiento. Estos datos deberían ser la base para estimular a las autoridades políticas a trabajar conjunta y coordinadamente con todos los actores y centros a nivel regional para dar un paso más y poder llegar a una mayor de población en riesgo. Los autores de este estudio han demostrado que se puede implementar un protocolo de actuación en el IAMEST e ir mejorándolo tras registrar e identificar los campos de mejora. Si se trabaja de forma coordinada globalmente, con toda seguridad no se tendrá que esperar otros 10 años para obtener frutos.

Como líneas de futuro, cabe plantearse la superespecialización de algunos centros para el tratamiento del shock cardiogénico secundario a infarto de miocardio. Esta entidad presenta un pronóstico ominoso (40\%$50 \%$ mortalidad a los 30 d) y desgraciadamente no existen hasta la actualidad medidas terapéuticas que hayan demostrado ser eficaces más allá de la propia revascularización precoz. Existen diferentes ensayos aleatorizados en marcha, con el uso de dispositivos de soporte ventricular, $(8,9)$ que podrán dar luz sobre su beneficio. En cualquier caso, la experiencia con estos dispositivos debería concentrarse en centros de alto volumen y diseñar un circuito específico para el transporte inmediato de los pacientes en shock cardiogénico (código shock).

\section{BIBLIOGRAFÍA}

1. Keeley EC, Boura JA, Grines CL. Comparison of primary and facilitated percutaneous coronary interventions for ST-elevation myocardial infarction: quantitative review of randomised trials. Lancet. 2006;367:579-88. https://doi.org/10.1016/S0140-6736(06)68148-8 2. Boersma E. Primary Coronary Angioplasty vs. Thrombolysis Group. Does time matter? A pooled analysis of randomized clinical trials comparing primary percutaneous coronary intervention and in-hospital fibrinolysis in acute myocardial infarction patients. Eur Heart J 2006;27:779-88. https://doi.org/10.1093/eurheartj/ehi810

3. Neumann FJ, Sousa-Uva M, Ahlsson A, Alfonso F, Banning AP, Benedetto U, et al; ESC Scientific Document Group. 18 ESC/ EACTS Guidelines on myocardial revascularization. Eur Heart J 2019; 40:87-165. https://doi.org/10.1093/eurheartj/ehy855

4. Regueiro A, Bosch J, Martín-Yuste V, Rosas A, Faixedas MT, GómezHospital JA, et al. Cost-effectiveness of a European ST-segment elevation myocardial infarction network: results from the Catalan Codi Infart network. BMJ Open. 2015;5(12):e009148. https://doi. org/10.1136/bmjopen-2015-009148

5. Kristensen SD, Laut KG, Kaifoszova Z, Widimsky P. Variable penetration of primary angioplasty in Europe -what determines the implementation rate? EuroIntervention. 2012;8 Suppl P:P18-26. https://doi.org/10.4244/EIJV8SPA5

6. Regueiro A, Rosas A, Kaifoszova Z, Faixedas MT, Curos A, Tresserras R, Sabaté M. Impact of the"ACT NOW. SAVE A LIFE"public awareness campaign on the performance of a European STEMI network. Int J Cardiol. 2015 Oct 15;197:110-2. https://doi.org/10.1016/j. ijcard.2015.06.040

7. Blanco F, Szarfer J, García Escudero A, Blanco R, Albornoz F, Alonso A, y cols. Detección de barreras e implementación de procedimientos para reducir la demora en el tratamiento del síndrome coronario agudo con elevación del segmento ST mediante angioplastia primaria. Experiencia de 20 años de un centro de referencia en una ciudad de alta densidad demográfica. Rev Arg Cardiol 2021;89:107-14.

8. Banning AS, Adriaenssens T, Berry C, Bogaerts K, Erglis A, Distelmaier K, et al; Collaborators. Veno-arterial extracorporeal membrane oxygenation (ECMO) in patients with cardiogenic shock: rationale and design of the randomised, multicentre, open-label EURO SHOCK trial. EuroIntervention. 2021;16(15):e1227-e1236. https://doi.org/10.4244/EIJ-D-20-01076

9. Udesen NJ, Møller JE, Lindholm MG, Eiskjær H, Schäfer A, Werner N, et al; DanGer Shock investigators. Rationale and design of DanGer shock: Danish-German cardiogenic shock trial. Am Heart J. 2019;214:60-8. https://doi.org/10.1016/j.ahj.2019.04.019 